CARTA AL EDITOR

Rev Chil Salud Pública 2019,

Vol 23(2): 174-175
Gilberto Bastidas Departamento de Salud Pública y Centro de Investigaciones Médicas y Biotecnológicas, Facultad de Ciencias de la Salud, Universidad de Carabobo.

bastidasprotozoo@hotmail.com

Tadeo Medina Ligia Gamez

Departamento de Salud Pública, Facultad de Ciencias de la Salud, Universidad de Carabobo.

Mariely Ramos Departamento de Salud Mental, Facultad de Ciencias de la Salud. Universidad de Carabobo.

\section{ENFOQUE ÉTICO EN LA SALUD PÚBLICA}

\author{
AN ETHICAL APPROACH TO PUBLIC HEALTH
}

Estimado editor

Las siempre complejas relaciones humanas en el diario quehacer, los indetenibles avances científico-tecnológicos y la innegable reducción presupuestaria en el sector salud requiere de la observación de la dimensión valorativa, la únicamente proveniente de los principios éticos. Se trata de una perspectiva normativa para la racional toma de decisiones en pro de la salud de todos aquellos individuos que conforman un grupo, de acuerdo con la definición que hiciera Ferrer ${ }^{1}$ sobre "ethos" al referirse a la misma como esfuerzo activo individual para dar forma humana a lo recibido pasivamente y transformarla en un esfuerzo colectivo para esculpir el bienestar de todos. ${ }^{2,3}$

Máxime porque en la práctica a la salud pública se le exige el logro de la salud de la comunidad sin detrimento de los derechos de las personas dentro de la misma; que en el desarrollo de programas y políticas sanitarias se incluyan los aportes de los miembros de la comunidad con especial empoderamiento de los marginados; que se garantice el acceso a la salud a todos los individuos de la comunidad; que en apego a la responsabilidad se obtenga la información necesaria y fidedigna para la implementación de programas sanitarios efectivos; que la actuación sea oportuna y que involucre distintos enfoques en procura de respetar las diversas creencias y culturas de los miembros de una comunidad; que resguarde la confidencialidad de la información que pueda dañar a individuo o comunidad; y por último, pero no menos importante, que no se abuse del poder durante la prestación de la atención de salud. ${ }^{4}$

Además la ética en salud colectiva es un paradigma que hoy día requiere de análisis en virtud de los avances tecnológicos en materia de tratamiento de las dolencias humanas, del diseño de nuevas modalidades de prestación de los servicios de salud y de la investigación en mujeres y hombres, que hace tiempo diera origen a lo que Potter3 llamara bioética en su intento por evitar los desmanes o perjuicios sobre el ser humano, pero que por estar el concepto enmarcado en la faceta de la ética clínica no parece suficiente para lograr el bien de las masas. De allí que se geste, o estén aplicándose principios éticos (criterios generales) en el diseño e implementación interdisciplinario de planes, políticas y programas socio-sanitarios, en lo que bien puede llamarse la ética en la salud pública. ${ }^{2}$

Esto resulta particularmente interesante porque la salud pública está inmersa en un elevado coeficiente de incertidumbre por no tener un rostro visible, concreto, sino por ser abstracta, pero innegablemente tangible a pesar de lo paradójico que parece. Por tanto, la toma de decisiones en materia de salud colectiva requiere de la revisión de los principios éticos principalistas (sustentada en valores como la no-maleficencia, beneficencia, autonomía y la justicia), dado que el manejo de las políticas en salud pública implica ineludiblemente la administración de riesgos y de complicaciones en grandes magnitudes y con elevada dificultad de predicción que limitan la aplicación del modelo bioético clínico en el cuidado de la salud colectiva. ${ }^{5,6}$

La salud pública muestra demora con respecto a otras disciplinas (porque la salud pública se ha considerado ética en sí misma y por su origen netamente vinculado al absolutismo ilustrado) en lo referente al determinante campo de la aplicación de la ética como punto de referencia único y clave para el análisis y discusión de las situaciones y contextos donde entran en conflicto los valores colectivos (si así pueden definirse) con los de corte individual y que, puede traducirse en incremen- 
to del riesgo a enfermar que tienen las poblaciones cuando se desconoce la existencia de los mismos o simplemente no se aplican.

El aparente estancamiento en el desarrollo e implementación de la ética en salud pública, a pesar del notable avance técnico-científico en el campo de la salud, la imparable transición epidemiológica (caracterizada por predominio de las enfermedades crónicas y poblaciones más viejas), la mayor demanda de servicios de atención en salud y la emergencia de los problemas éticos de carácter global, exigen en primer lugar que se retome y en segundo lugar que se profundicen los estudios sobre la ética en salud colectiva dada su relevancia social en procura del bienestar.6,7

Entonces se han propuesto algunos principios éticos para dar respuesta (como guías generales de acción) a aspectos de la salud colectiva como: la restricción a la libertad individual en epidemias infecciosas (en relación con limitaciones del tránsito, aislamiento, segregación, cuarentena e inmunizaciones); límites de la intervención en la promoción de estilos de vida; destino del presupuesto para la salud colectiva; consentimiento informado en la población en intervenciones en salud masivas y nivel de riesgo socialmente aceptable; investigaciones en poblaciones vulnerables; $y$ en la responsabilidad internacional de los países frente a pandemias. ${ }^{8}$

Con miras a combatir las inequidades en salud y mejorar la calidad de vida de los grupos sociales se señalan como principios éticos en salud pública a la equidad (en relación a paridad en oportunidades y trato), la solidaridad (colaboración y ayuda mutua para la obtención del bienestar común, tiene implícita la igualdad), la responsabilidad (cuidado de la población en riesgo o vulnerable a través del diseño e implementación de planes, políticas y programas de salud, por medio de los órganos socio-sanitarios oficiales) y la protección (actitud de resguardo o cobertura de necesidades que se convierte en un compromiso irrenunciable una vez que es asumido libremente, sin confundirse con el paternalismo beneficente). ${ }^{9-11}$

En conclusión la salud pública como esfuerzo o arte colectivo para promover la salud y prevenir las enfermedades, dentro de cualquier sistema político-administrativo, debe valerse de principios éticos en la resolución de los dilemas que sin ninguna duda se presentan en autoridades sanitarias o en la propia población objeto de la intervención en salud, porque el ámbito de acción de la salud pública va mas allá de la aplicación de estrictas políticas sanitarias, pues debe combatir la inequidad en salud biológica y socialmente evitables en plena consideración de la ponderada reflexión valorativa en la toma de decisiones.

\section{REFERENCIAS BIBLIOGRÁFICAS}

1. Ferrer J. Introducción a los paradigmas teóricos en bioética. Apuntes para el Master Universitario en Bioética. Universidad Pontificia Comillas; Madrid, España, 1997.

2. Oliver-Vásquez $M$. La ética de la salud pública. $P$ R Health Sci J, 2003; 2(1): 21-22.

3. Potter V. Bioethics bridge to the future. Prebtis Hall, New York, 1971.

4. Public Health Leadership Society. Principles of the ethical practice of public health. Version 2.2. [internet], 2001 [Citado el 07.01.2019]. Disponible en: https://www.apha.org/-/media/files/pdf/membergroups/ ethics/ethics_brochure.ashx

5. Comisión Nacional para la Protección de los Sujetos Humanos. De investigación biomédica y del comportamiento. El informe Elmont. Principios y guías éticos para la protección de los sujetos humanos de investigación. [internet], 1979 [Citado el 20.11.2018]. Disponible en: http://www.bioeticayderecho.ub.edu/ archivos/norm/InformeBelmont.pdf.

6. Beauchamp T, Childress J. Principles of Biomedical Ethics. 4t h Ed. Oxford University Press, Oxford, 1994.

7. Puyol A. Ética y salud pública. Dilemata, 2014; 6(15): 15-22.

8. Kottow M. Bioética en Salud Pública. Editorial Puerto de Palos, Santiago de Chile, 2005.

9. Ascurra M, Rosales C. Formación y capacitación de recursos humanos de la bioética en salud pública. En Málaga, H. (Compilador) Salud Pública. Enfoque Bioético. Editotial Dislimer, Caracas, 2005.

10. Rodríguez M, Urbanos R (eds.). Desigualdades sociales en salud. Masson, Barcelona, 2008.

11. Lolas F. El Desafío Bioético de la equidad: Su relevancia en salud pública. Rev Esp Salud Pública, 2001; 75(3): 187-192. 